\title{
Photo Thermal Generator of Selective Radiation Structural and Energetic Features
}

\author{
A. M. Kasimakhunova ${ }^{1 *}$, Sh. A. Olimov ${ }^{2 *}$, L. K. Mamadalieva ${ }^{1}$, M. Norbutaev $^{1}$, Sh. S. Nazirjanova1, \\ Sajida Riffat Laraib ${ }^{2,3^{*}}$
}

${ }^{1}$ Fergana Polytechnic Institute, Fergana, Uzbekistan

${ }^{2}$ School of Renewable Energy, North China Electric Power University, Beijing, China

${ }^{3}$ National Engineering Laboratory for Biomass Power Generation Equipment, Beijing, China

Email: ^kasimahunova@rambler.ru, ^shoirbekolimov@yandex.ru, ^sajida.laraib@gmail.com

How to cite this paper: Kasimakhunova, A.M., Olimov, Sh.A., Mamadalieva, L.K., Norbutaev, M., Nazirjanova, Sh.S. and Laraib, S.R. (2019) Photo Thermal Generator of Selective Radiation Structural and Energetic Features. Journal of Applied Mathematics and Physics, 7, 1263-1271. https://doi.org/10.4236/jamp.2019.76086

Received: May 5, 2019

Accepted: June 24, 2019

Published: June 27, 2019

Copyright (c) 2019 by author(s) and Scientific Research Publishing Inc. This work is licensed under the Creative Commons Attribution International License (CC BY 4.0).

http://creativecommons.org/licenses/by/4.0/

\begin{abstract}
Photo thermoelectric generators (PTEGs) are solid state heat engines that generate electricity from concentrated sunlight. In this paper, we developed a novel detailed balance model for PTEGs and applied this model to both state-of-the-art and idealized materials. This model uses thermoelectric compatibility theory to provide analytic solutions to device efficiency in idealized materials with temperature-dependent properties. The method for the theoretical calculation of the mechanism of absorption and refraction of light radiation of a selected wavelength in the volume of a photoelectric converter and the design of an effective photo thermal converter with a fixed slit are presented in the article. Direct- and non-direct-electron transitions from the valence band to the conduction band are analyzed. The possibility of optimal distribution of the light spectrum on the frontal surfaces of the solar cell and thermoelectric converter is shown. According to the results of experimental studies, a conclusion was made that the efficiency of a photo converter increases by a factor of three as compared with analogues, but operating without a thermal converter.
\end{abstract}

\section{Keywords}

Photo Converter, Thermoelectric Converter, Selective Radiation, Solid State Heat En-Gines

\section{Introduction}

A solid-state device known as thermoelectric power, based on Seebeck effect, provides direct energy conversion from thermal energy into electrical energy due to temperature gradient. The thermoelectric power cycle, with charge carriers 
resembles the power cycle of a conventional heat engine. Thermoelectric power generators offer several distinct advantages over other technologies [1] [2] [3] [4]. Systems based on it have been developed and studied for many years. When a temperature difference is established between the hot and cold junctions of two dissimilar materials (metals or semiconductors), a voltage is generated, i.e., Seebeck voltage. In fact, this phenomenon is applied to thermocouples that are extensively used for temperature measurements. A thermoelectric device creates voltage when there is a different temperature on each side. Conversely, when a voltage is applied to it, it creates a temperature difference [5].

The idea of creating and primarily studying a highly efficient photo thermo generators (FTG) of a selective radiation was justified [6] as the main essence of the invention was to simplify the transformation of energy, product construction and to eliminate the additional costs. However, there are still a number of problems associated with the location, as well as a clear orientation of the individual converters along the optical axis of light, the likelihood of increased radiation losses when it is separated by spectral composition and blocking of the converter design due to the need to add optical devices. In this regard, the results of the development and initial studies of the electrophysical and energetic characteristics of the convertor with a selected gap are presented in this article.

This is due to the fact that all these studies are accompanied by the complication of the technology of manufacturing solar cells, the need for expensive equipment such as vacuum systems, electron microscopes and annealing furnaces, etc. In addition, all recommended technologies require consumption of various types of energy resources that include both the electrical and fuel. Photovoltaics require solar cells (SE) that do not require unnecessary costs, bulky equipment and are energy-efficient. However, despite the efficiency given off by the combination of photovoltaics and thermoelectric converters (TEC) by converting solar energy into electric energy, there were two inevitable problems. First is that there is a strong dependence on the temperature of the output useful power of a solar cell that's at the spectral composition of the incident radiation and second is the negative dependence of the same power on the temperature of the element. Further research led to the creation of a selective radiation photo-thermal generator, which provided for the illumination of the surface of a solar cell with only photoactive radiation. However, the thermoelectric compatibility theory has not been widely researched to provide analytic solutions of device efficiency in idealized materials with temperature-dependent properties.

The technical solution required solving several problems related to the location as well as the precise orientation of the converters on the optical axis of light, the likelihood of increasing radiation losses due to the division of the spectral composition, and problems related to the cluttering design of the transducers. Optical devices were added to cater to the problems.

Therefore, we developed an optimal design of a highly efficient photothermogenerator (FTG) of selective radiation and studied the physics of absorption 
and refraction of light in the photovoltaic part of it. In this connection, in the present work, the results of theoretical researches, (theoretical studies) of development and initial tests of electrophysical and energetic characteristics of FTG with the chosen slit are presented. This work is essentially a continuation of work [2]. Therefore, this article gives the method for the theoretical calculation of the mechanism of absorption and refraction of light radiation of a selected wavelength a photoelectric converter and the design of an effective photo thermal converter with a fixed slit, also direct-and non-direct-electron transitions from the valence band to the conduction band were analyzed to minimize the loses.

\section{Calculation and Study of the Distribution of Electromagnetic Radiation}

Comparison and analysis of the electrical properties of semiconductors and metals show their proximity. However, with dielectrics they are very different and therefore, the spreading of a light beam radiation in a semiconducting substance, at the absence of external fields, can be described by solving the Maxwell equation [7]

$$
\left.\begin{array}{ll}
\operatorname{rot}(E)=-\mu_{0} \mu \frac{\mathrm{d} H}{\mathrm{~d} t}, & \operatorname{div} E=0 \\
\operatorname{rot}(H)=\varepsilon_{0} \varepsilon_{1} \frac{\mathrm{d} E}{\mathrm{~d} t}+\sigma E, & \operatorname{div} H=0
\end{array}\right\}
$$

where $E$ and $H$ is the intensity of electric and magnetic fields, respectively, $\varepsilon_{0}$ is the dielectric penetration co-efficient of a semiconductor and $\mu_{0}$ is its magnetic penetration coefficient in vacuum.

In formula (1), it can be noted that in the second equation of the system the conduction current density $j=\sigma E$. was taken into account. Since it is written in the international system of units (SU), the parameters are taken with the following values: $\sigma_{0}=\left(4 \pi \times 9 \times 10^{9}\right)^{-1} \mathrm{f} / \mathrm{m} ; \mu_{0}=4 \pi \times 10^{-7} \mathrm{gn} / \mathrm{m}$. The dielectric and magnetic penetration coefficients of a semiconductor are functions of the frequency $\omega\left(\varepsilon_{1}(\omega)\right.$ and $\left.\mu_{1}(\omega)\right)$. They are anisotropic and are represented by second-rank tensors. To study optical phenomena in semiconductor crystals, we assume that the external field is absent and the influence of the electromagnetic wave field is very weak. In this case, $\sigma, \varepsilon_{1}$ and $\mu$ become scalar values. Based on the fact that

$$
\operatorname{rot} \frac{\mathrm{d} H}{\mathrm{~d} t}=\frac{\mathrm{d}}{\mathrm{d} t} \operatorname{rot} H
$$

and, after some mathematical operations, we can have an equation in the form

$$
\nabla^{2} E=\Delta E=\mu \mu_{0} \sigma \frac{\mathrm{d} E}{\mathrm{~d} t}+\mu \varepsilon_{1} \mu_{0} \varepsilon_{0} \frac{\mathrm{d}^{2} E}{\mathrm{~d} t^{2}}
$$

In formula (3) it is assumed $\operatorname{rotrot} E=\operatorname{grad} \operatorname{div} E-\nabla^{2} E$, and $\operatorname{grad} \operatorname{div} E=0$. Thus, we can have several solutions for (3). The most acceptable and appropriate solution of (3) is to obtain an equation for the intensity of the electric field in the form 


$$
E=E_{0} \exp i \omega\left(t-\frac{z}{v}\right) .
$$

and this expression is a wave spreading in the $z$ direction with the velocity $v$ and angular frequency $\omega$. [8]. The same can be obtained for the magnetic field strength. In order to obtain (4) as the final equation it is necessary to fulfill the conditions of (3) corresponding to the integrated complex index of refraction

$$
v^{-2}=\mu \varepsilon_{1} \mu_{0} \varepsilon_{0}-i \frac{\sigma \mu_{0} \mu}{\omega}
$$

In general, to satisfy Equation (4), it is better to take into account that firstly, the square of the propagation speed of light in a vacuum with $c^{2}=\left(\mu_{0} \varepsilon_{0}\right)^{-1}$ and secondly, in the optical range of most semiconductors, in particular silicon, the magnetic properties are weak. It can be taken as $\mu \approx 1$. Also, taking into account the rate between the refractive index and the absorption index and the dielectric constant, the specific electrical conductivity can be written as

$$
\varepsilon=(n-i k)^{2}=\varepsilon_{1}-i \frac{\sigma}{\varepsilon_{0} \omega}=\varepsilon_{1}-i \varepsilon_{2}
$$

In (6) the speed of light spreading is taken $c^{2}=\left(\mu_{0} \varepsilon_{0}\right)^{-1}$. The expression for complex refraction, after separation of the real and imaginary parts, can be written in the following form of the system of equations

$$
\left.\begin{array}{l}
n^{2}-k^{2}=\varepsilon_{1}(\omega), \\
2 n k=\frac{\sigma}{\varepsilon_{0} \omega}=\varepsilon_{2}(\omega) .
\end{array}\right\}
$$

Here $\varepsilon(\omega)$ is the complex dielectric constant, $\varepsilon_{1}(\omega)$ is the real part, and $\varepsilon_{2}(\omega)$ is the coefficient of the imaginary part.

On the one hand, the rate between the complex refractive index and (6), as well as the dielectric permittivity on the other hand allows us to conclude that the optical constants of the substance are equivalent in their generality. This covers the macroscopic parameters characterizing the interaction of the electromagnetic wave and the absorbing medium.

$$
n_{*}=n-i k
$$

It was shown that, based on the causality condition, one can write formulas connecting $n$ and $k$ with each other [9]

$$
\begin{aligned}
& n=1+\frac{2}{\pi} \int_{0}^{\infty} \frac{\omega k}{\omega^{2}-\omega_{0}^{2}} \mathrm{~d} \omega \\
& k=-\frac{2 \omega_{0}}{\pi} \int_{0}^{\infty} \frac{n}{\omega^{2}-\omega_{0}^{2}} \mathrm{~d} \omega .
\end{aligned}
$$

The Equation (9) in our case is basic and important, because according to this expression $n$ can be calculated at any frequency $\omega_{0}$ in the interval from zero to infinity, and therefore, based on the selected selective absorption spectrum, the spectrum of the refractive index can be calculated and vice versa. So, the above method allows us to write relationships that connect $n^{2}-k^{2}=\varepsilon_{1}(\omega)$ and $2 n k=\varepsilon_{2}(\omega)$ : 


$$
\begin{aligned}
& n_{1}\left(\omega_{0}\right)=\left(n^{2}-k^{2}\right)_{\omega_{0}}=1+\frac{2}{\pi} \int_{0}^{\infty} \frac{\omega \varepsilon_{2}(\omega)}{\omega^{2}-\omega_{0}^{2}} \mathrm{~d} \omega, \\
& \varepsilon_{2}\left(\omega_{0}\right)=(2 n k)_{\omega_{0}}=-\frac{2 \omega_{0}}{\pi} \int_{0}^{\infty} \frac{\omega \varepsilon_{2}(\omega)}{\omega^{2}-\omega_{0}^{2}} \mathrm{~d} \omega,
\end{aligned}
$$

Thus, to the wave Equation (4) substituting (5) and (8) it turns out

$$
E=E_{0} \exp \left(-\frac{\omega k z}{c}\right) \exp i \omega\left(t \frac{n z}{c}\right) \text {. }
$$

This shows that the main absorption coefficient $k$ characterizes the attenuation of an electromagnetic wave in a semiconductor.

As for the semiconductor band structure, for a start, we can consider the simplest band structure. It is a spherical non-degenerate and energetic surface for electrons and holes with a quadratic dispersion law. Analytically such an energy structure can be expressed by the equation

$$
\left(E-\frac{\hbar^{2} k^{2}}{2 m_{n}}\right)\left(E+E_{g}+\frac{\hbar^{2} k^{2}}{2 m_{p}}\right)=0 .
$$

where $\hbar$ is Planck's constant divided by $2 \pi$. The value of $\hbar=1.05 \times 10^{-34} \mathrm{~J} \cdot \mathrm{sec}$.

The roots of Equation (14) are

$$
E_{c}=\frac{\hbar^{2} k^{2}}{2 m_{n}} ; E_{v}=-E_{g}-\frac{\hbar^{2} k^{2}}{2 m_{p}} ;
$$

However, the silicon diagram of the conduction band differs from the simplest band structure [10] [11]. The difference in band structures exists even with germanium. In silicon, the absolute minimum is located at a different brillouin zone in accordance with a different number of ellipsoids (valleys) in the zone. In addition, the quantitative rates between the energies at some points are significantly different.

The dependences of the energy on the wave number near the absolute minimum for the case when the coordinate axes coincide with the direction of the main axes of the mass ellipsoids for multi-valley semiconductors can be represented as

$$
E(k)=\frac{\hbar^{2}}{2}\left(\frac{k_{x}^{2}}{m_{l}}+\frac{k_{y}^{2}+k_{z}^{2}}{m_{t}}\right),
$$

where $m_{l}$ and $m_{t}$ are the longitudinal and transverse components of the mass ellipsoid.

\section{The Design of a Selective Photo Thermo Generator with a Fixed Slit Trench}

Selective photo thermo generator given in [1] energy performance exceeds FTG with equal numbers of cascades [12]. However, when using them as power plants of even low power, there are some problems associated with cluttering up their overall structure. Because the presence of lenses, other optical glasses and their attachment mechanisms, to some extent obscures the front surface of con- 
verters. In addition, the choice of photoactive radiation from the total illumination does not guarantee a clear distribution of light exactly into two parts, which without difficulty will be possible to direct to the surface of the photoconverter and the thermal converter.

For a positive solution to this problem, design of a photo thermo generator with a fixed gap was designed by us (Figure 1).

\section{Optical Diagram of the Position Selective Photoconverter}

This design provides for the arrangement of silicon photoelectric and thermoelectric converters based on semiconductor materials from the ternary compounds BiTeSe (n-type) and BiTeSb (p-type) so that, depending on the photo-activity of the FEP on a certain radiation spectrum, after the dispersion of the light beam into the optical an axis is placed on a photoconverter, then a TEP is mounted around the FEP. This method of installation and connection is almost always necessary. This is explained by the following. First, the light passing through the optical system distributes the color sequence based on the electrical and optical properties of the material of the optical glass, and secondly, there are still no specific research results recommending highly efficient separation of light into two parts; Often the required spectrum may be in the middle of divided colors

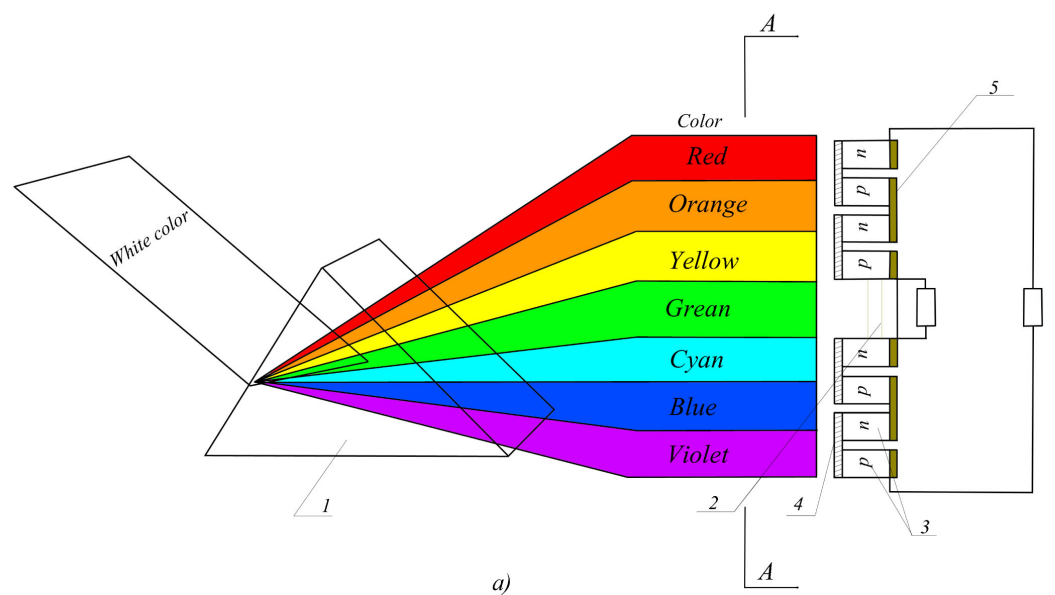

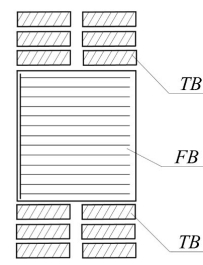

Viev A-A. I-Option

b)

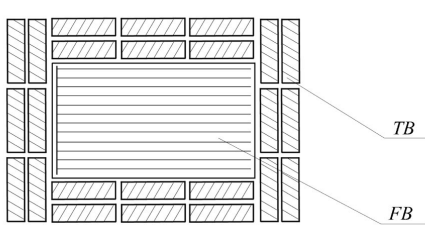

Viev A-A. II-Option

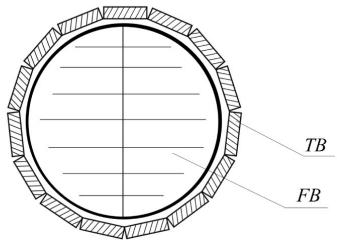

Viev A-A. III-Option

d)

Figure 1. Design of a photo thermo generator with a fixed gap. a) 1-prism, 2-photoelectrical converter (PhC), 2-p- and n-branches of thermo converter (ThC), 3-hot switching plates (TEP), 4-cold switching plates of (TEP), b, c, d: Accordingly, three variants of arrangement of (FB) and (TB) on section A-a: I, II, III-Variant. 
Thirdly, a solar cell made of the same semiconductor material with different technologies has a spectral sensitivity in different color areas [13]. Fourth, the solar cell is not always made of silicon. It can be made of other semiconductor materials both by homo and heterostructure. It can be seen from the figure that the previously measured and determined photoactive radiation through a special slit of increasing light loss, the slit through which the selected radiation passes, must be strictly in terms of the geometric dimensions of the FEP surface. A thermoelectric converter is placed around the photoelectric converter, depending on the location of other radiations [14]. In general, it is possible to assemble both converters on the same plane. At the same time, according to the results of theoretical studies, the coefficient of absorption and refraction will obey the well-known laws of physics.

The front surfaces of the converter can be assembled with different configurations (see Figure 1, I, II, III options). It depends on the geometric shape of the photoelectric converter and the size of the area of the divided light spot [15]. Attaching the switching plates of the hot junctions of the thermocouple into one single ring-shaped or right-angled common surface (for example, from the beryllium oxide material $\mathrm{BeO}$, since the temperature coefficients of expansion of $\mathrm{BeO}$ and the hot switching plates of TE are close).

\section{Experimental Study}

Testing the practicality of using a selective photo thermo generator of light radiation showed that the introduction of this design will lead to simplification of assembly and assembly work in the construction of power plants. The loss of light energy due to the space between the converters is about 3\% (for the case of covering the surface of a thermal converter of a continuous heat-receiving $\mathrm{BeO}$ ). The efficiency of a thermoelectric converter is almost equal to the values given in domestic and foreign literature [16], that is, when the temperature of the cold junction is equal to the ambient temperature $T_{X}=20^{\circ} \mathrm{C}$ and hot junction $T_{F}=$ $1200 \mathrm{C} \eta=6.0 \%$. The temperature of the photoconverter is practically unchanged [17]. When using twenty-fold solar radiation, it rose only 1.10C. This can apparently be explained by the error and measurement of the light intensity during photometry and the lack of a clear boundary between the spectral wavelengths.

\section{Conclusion}

Based on the scientific and theoretical design decisions, it can be concluded that the photo thermoelectric converter of light energy into electrical energy with a fixed slit trench is one of the original technical solutions in the use of alternative sources of electrical energy. It allows to use photo converter and thermo converter from a semiconductor of any structure with least radiation losses and prevention of blockage makes it an efficient energy source. It also would lead to simplification of assembly and its work in the construction of power plants. Of course, the use of relatively efficient converters leads to the most tangible results. 


\section{Acknowledgements}

The author would like to acknowledge Mr. Muhammad Ibrahim and Mrs. Riffat Tahira for their guidance and support, also, would like to thank Miss Nayab Fatima Noor for technical support.

\section{Conflicts of Interest}

The authors declare no conflicts of interest regarding the publication of this paper.

\section{References}

[1] Riffat, S.B. and Ma, X. (2003) Thermoelectrics: A Review of Present and Potential Applications. Applied Thermal Engineering, 23, 913-935. https://doi.org/10.1016/S1359-4311(03)00012-7

[2] Omer, S.A. and Infield, D.G. (2000) Design and Thermal Analysis of Two Stage Solar Concentrator for Combined Heat and Thermoelectric Power Generation. Energy Conversion \& Management, 41, 737-756. https://doi.org/10.1016/S0196-8904(99)00134-X

[3] Yadav, A., Pipe, K.P. and Shtein, M. (2008) Fiber-Based Flexible Thermoelectric Power Generator. Journal of Power Sources, 175, 909-913. https://doi.org/10.1016/j.jpowsour.2007.09.096

[4] Jinushi, T., Okahara, M., Ishijima, Z., Shikata, H. and Kambe, M. (2007) Development of the High-Performance Thermoelectric Modules for High Temperature Heat Sources. Materials Science Forum, 534-536, 1521-1524. https://doi.org/10.4028/www.scientific.net/MSF.534-536.1521

[5] Patel, M.D., Mehta, P.S.B. and Shah, M.P. (2015) Review of Thermoelectricity to Improve Energy Quality. International Journal of Emerging Technologies and Innovative Research, 2, 847-850. https://www.jetir.org

[6] Kasimakhunova, A.M., Olimov, S., Nurdinova, R., Iqbal, T. and Mamadalieva, L.K. (2018) Highly Efficient Conversion of Solar Energy by the Photoelectric Converter and a Thermoelectric Converter. Journal of Applied Mathematics and Physics, 6 , 520-529. http://www.scirp.org/journal/jampUSA

[7] Jiang, B.-N., Wu, J. and Povinelli, L.A. (1996) The Origin of Spurious Solutions in Computational Electromagnetics. Journal of Computational Physics, 125, 104-123. https://doi.org/10.1006/jcph.1996.0082

[8] Mao, F., Yao, J., Zhou, Y., Dong, C., Kursten, B. and Macdonald, D.D. (2019) Determining the Electric-Field Strength in a Passive Film via Photo-Induced Electric Fields. Corrosion Science, 154, 239-245.

[9] Moss, T.S. and Hawkins, T.D. (1958) Low-Level Absorption in Germanium. Physical Review Letters, 1, 129. https://doi.org/10.1103/PhysRevLett.1.129

[10] https://bipm.org/utils/common/pdf/CGPM-2018/26th-CGPM-Resolutions.pdf

[11] Kasimakhunova, A., Olimov, S., Nurdinova, R., Iqbal, T. and Mamadalieva, L. (2018) Highly Efficient Conversion of Solar Energy by the Photoelectric Converter and a Thermoelectric Converter. Journal of Applied Mathematics and Physics, 6, 520-529. https://doi.org/10.4236/jamp.2018.63047

[12] Su, Y., Jiang, Y., Wu, Z. and Zhao, G. (2011) Spectral Response of Metal-Semiconductor-Metal Photodetector Based on Black Silicon. Energy Procedia, 12, 615-619. https://doi.org/10.1016/j.egypro.2011.10.083 
[13] Meija, J., Coplen, T., Berglund, M., et al. (2016) Atomic Weights of the Elements 2013 (IUPAC Technical Report). Pure and Applied Chemistry, 88, 265-291. https://doi.org/10.1515/pac-2015-0305

[14] Zaynabidinov, S., Aliev, R., Muydinova, M. and Urmanov, B. (2018) On the Optical Efficiency of Silicon Photoelectric Converters of Solar Energy. Applied Solar Energy, 54, 395-399. https://doi.org/10.3103/S0003701X1806018X

[15] Ting, C.-C. and Chao, W.-S. (2010) Measuring Temperature Dependence of Photoelectric Conversion Efficiency with Dye-Sensitized Solar Cells. Measurement, 43, 1623-1627. https://doi.org/10.1016/j.measurement.2010.09.025

[16] Gupta, P.N. (1987) Propagation of Electromagnetic Waves in Semiconductor Plasma under the Influence of Crossed Electric and Magnetic Fields. IETE Journal of Research, 33, 36-37. https://doi.org/10.1080/03772063.1987.11436654

[17] Chen, J., Li, K., Liu, C., Li, M., Lv, Y., Jia, L. and Jiang, S. (2017) Enhanced Efficiency of Thermoelectric Generator by Optimizing Mechanical and Electrical Structures. Energies, 10, 1329. https://doi.org/10.3390/en10091329 